\title{
Video modeling para enseñar habilidades sociales a niños con trastorno del espectro autista. Una revisión sistemática
}

\author{
Video modeling to teach social skills to children with autism spectrum \\ disorder. A systematic review
}

\begin{abstract}
Resumen
La eficacia del video modeling como herramienta educativa para personas con autismo ha demostrado ser un área prometedora para investigadores y miembros de la comunidad educativa. Durante las tres últimas décadas, la literatura científica ha mostrado la utilidad y eficiencia del video modeling para enseñar una variedad de conductas distintas. El objetivo del trabajo es realizar una revisión sistemática de aquellos estudios que han aplicado el video modeling como método para enseñar habilidades sociales; dichos estudios se han clasificado en función del tipo de diseño que utilizan, las características de la muestra, el tipo de intervención (si aplican video modeling solo o en combinación con otras técnicas) y los resultados obtenidos. Se han realizado resúmenes descriptivos de cada estudio y análisis de los resultados. En las conclusiones se indican pautas para la aplicación del video modeling en futuras intervenciones.
\end{abstract}

\section{Palabras clave}

Trastornos del espectro autista, video modeling, habilidades sociales, intervención educativa.

\begin{abstract}
The effectiveness of video modeling as an educational tool for people with autism has proven to be a promising area for researchers and members of the educational community. During the last three decades, scientific literature has shown the usefulness and efficiency of video modeling to teach a variety of different behaviors. The objective of the work is to carry out a systematic review of those studies that have applied video modeling as a method to teach social skills. These studies have been classified according to the type of design they use, the characteristics of the sample, the type of intervention (if video modeling is applied alone or in combination with other techniques) and the results obtained. Descriptive summaries of each study and analysis of the results have been made. The conclusions indicate guidelines for the application of video modeling in future interventions.
\end{abstract}

\section{Keywords}

Autism spectrum disorders, video modeling, social skills, educational intervention.

\section{Thais Rosal Negre}

<thaisrn@blanquerna.url.edu>

Universidad Ramon Llull. España

\section{Ignasi Ivern Pascual}

<ignasiip@blanquerna.url.edu>

Universidad Ramon Llull. España

\section{Climent Giné Giné}

<climentgg@blanquerna.url.edu> Universidad Ramon Llull. España

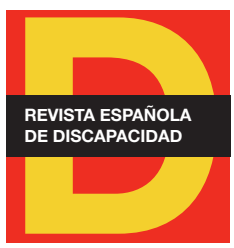

Para citar:

Rosal, T. et al. (2018): "Video modeling para enseñar habilidades sociales a niños con trastorno del espectro autista. Una revisión sistemática". Revista Española de Discapacidad, 6 (I): 31-47.

Doi: <https://doi.org/10.5569/23405104.06.01.02>

Fecha de recepción: 01-08-2017 Fecha de aceptación: 19-03-2018 


\section{Introducción}

El autismo es un trastorno grave del neurodesarrollo que afecta a la comunicación y al funcionamiento social y se caracteriza por la presencia de comportamientos repetitivos e intereses restringidos (American Psychiatric Association, 2013). Las personas con trastorno del espectro autista (TEA) plantean grandes retos a las familias, a la comunidad educativa y a la sociedad (Rivière y Núñez, 2008). Estos retos son, en primer lugar, de comprensión de las características del trastorno por parte de la sociedad; resulta difícil entender los procesos psicológicos internos de personas con graves problemas de comunicación y relación. Estos déficits, son considerados clave para el diagnóstico del TEA y afectan a una variedad de habilidades como pueden ser el juego, el uso del lenguaje social y las iniciaciones de interacciones sociales (Wilson, 2013b). En segundo lugar, de explicación; aún quedan por desvelar muchas cuestiones acerca de las causas de la génesis biológica y los procesos cognitivos de las personas con autismo. Por último, de educación; presentan dificultades de relación que dificultan los procesos normativos de aprendizaje: imitación, aprendizaje observacional e intercambio a nivel simbólico. Parece pues, de vital importancia, como afirma Delano (2007), poder identificar intervenciones educativas eficaces para este tipo de población.

Actualmente, existe evidencia científica sobre la importancia de la comunicación social como componente clave para mejorar la calidad de vida de las personas con TEA (Baron-Cohen et al., 1985; Bellini y Akullian, 2007; Koegel, et al., 2014). Baron-Cohen et al. (1985) afirman que existe una capacidad subyacente a la cognición social que consiste en atribuir estados mentales a los que nos rodean; nos permite saber (imaginar) lo que quieren, sienten o ven. Esta capacidad se conoce como Teoría de la Mente (TM) y resulta clave para poder desarrollar una buena comunicación que no se limite solo a hacer demandas sobre las propias necesidades (Koegel et al., 2014). La TM es decisiva para mejorar la calidad de vida de personas con TEA, la ausencia o retraso de la misma explica muchos aspectos de su comportamiento como, por ejemplo, la capacidad de compartir los estados emocionales y captar a los demás como seres dotados de intenciones es la base sobre la que se desarrolla la noción de que las acciones de los demás están guiadas por sus estados mentales. La TM nos permite predecir la conducta de los demás y dar significado al mundo que nos rodea, punto relevante en la sintomatología del trastorno (Perinat, 2007). Por lo tanto, y siguiendo con las explicaciones de Perinat (2007), el desarrollo cognitivo está asociado a la internalización de los significados sociales. La presencia de estas limitaciones en el área de la interacción social es una de las características típicas de este trastorno. Aunque no es la única. Parece pues que existe una relación directa entre la TM y las habilidades sociales (Newton y Jenvey, 2011). El escaso desarrollo de la primera, junto con el déficit de lenguaje funcional que suelen presentar los niños con TEA, puede explicar muchas dificultades de relación. Las habilidades sociales (HHSS en adelante) empiezan a desplegarse durante la primera infancia y, a lo largo del proceso de desarrollo iremos adquiriendo habilidades que en un futuro nos permitirán ser efectivos y adecuados en nuestras interacciones con los demás (Odom et al., 2008). Las dificultades que presentan las personas con TEA en los procesos de aprendizaje "normativos" como la imitación, el aprendizaje observacional o el juego provocan alteraciones en la competencia necesaria para que un niño o niña utilice de forma efectiva y adecuada comportamientos sociales para establecer con éxito relaciones entre iguales (Odom et al., 2006).

Hay que tener en cuenta que en las últimas décadas la prevalencia de TEA ha incrementado de forma significativa. Lejos están los índices de 4.5 / 10.000 (Baron-Cohen et al., 1985) que se aceptaban por la 
comunidad científica cuando hoy en día la CDC (Centers for Disease Control and Prevention) afirma que la prevalencia estimada de niños diagnosticados con TEA es de 1 de cada 68 (Christensen et al., 2016). Dentro del contínuum de las técnicas educativas adaptadas a este tipo de población, parece oportuno y necesario el uso de nuevos métodos educativos basados en la evidencia científica, centrados en estimular la cognición social y el lenguaje y que, en conjunto, sean útiles a los diferentes tipos de perfiles TEA existentes. De acuerdo con la literatura disponible, una alternativa eficaz se centra en trabajar estrategias que impliquen habilidades relacionadas con el juego social; a pesar de su complejidad, se pueden dividir los aspectos que las componen en determinadas conductas muy básicas que el niño o niña ha de aprender, como las iniciaciones verbales o motoras y la imitación (Sancho, et al., 2010). Durante los últimos años, una forma de enseñar habilidades relacionadas con el juego social es utilizar el video modeling (VM en adelante), que se ha revelado como técnica muy útil y eficiente para este tipo de población (Bellini y AkuIlian, 2007; Wang y Koyama, 2014), ya que permite enseñar una gran variedad de habilidades diferentes en un tiempo razonable de intervención. Como afirma Corbett (2003), el VM forma parte de las técnicas de modificación de conducta; ha sido desarrollado para facilitar el aprendizaje observacional. La metodología ABA (Applied Behaviour Analysis) dispone de evidencia científica (Corbett, 2003) y se ha consolidado como un método de enseñanza eficaz para el tratamiento de personas con TEA. Basándonos en la literatura disponible, una opción eficaz se centra en trabajar estrategias que impliquen habilidades relacionadas con el juego social. EI VM forma parte de este significativo número de métodos educativos basados en las técnicas de modificación de conducta.

En los últimos años ha surgido la necesidad de utilizar estrategias basadas en la evidencia científica para promover el desarrollo de los niños con TEA, así como para identificar estrategias basadas en la práctica científica. Ello ha dado como resultado una gama de prácticas que han demostrado su eficacia, entre ellas el VM (Bellini y Akullian, 2007). Estas investigaciones se han centrado tradicionalmente en aprendizajes funcionales y no tanto en temas más complejos como la competencia social. Como se ha comentado anteriormente, debido a que las personas con TEA presentan retraso en la adquisición de la TM, a menudo éstas manifiestan ansiedad frente a las conductas de los demás porque tienen muchas dificultades para predecir su comportamiento (Baron-cohen et al., 1985). En estudios anteriores se ha comprobado que las personas con TEA son capaces de aprender habilidades implicadas en el desarrollo de TM como perspectivas visuales, identificar una situación con un pensamiento o emoción...(Ozonoff y Miller, 1995), pero una de las limitaciones de estos estudios son los problemas que presenta este tipo de población para generalizar las conductas aprendidas. Existen revisiones que muestran que el VM es una práctica basada en la evidencia científica útil para enseñar una amplia gama de conductas, incluyendo las HHSS (Bellini y Akullian, 2007; Reichow y Volkmar, 2010). Por este motivo y como afirman Nikopoulos y NikopoulouSmyrni (2008), parece conveniente continuar investigando su eficacia en el ámbito de las HHSS donde la conducta a aprender es compleja e incluye una cadena de acciones que implican a otra persona además del propio participante.

Diversos metaanálisis y revisiones sistemáticas señalan que existen diferentes tipos de VM (Bellini y AkuIlian, 2007; Shukla-Mehta et al., 2010; Wang y Koyama, 2014): adult-VM (se utiliza un adulto para gravar la conducta objetivo), peer-VM (la conducta la realizan compañeros del participante) y Video Self Modeling (el participante es el protagonista del vídeo, donde se puede observar a sí mismo realizando la conducta a aprender de forma adaptativa porque el vídeo ha sido editado previamente para eliminar los errores). El Video Self Modeling es un método eficaz que cumple los criterios de calidad en investigación en educación 
especial descritos por Odom et al. (2005). También existe evidencia que apoya el VM como herramienta para enseñar habilidades sociales (HHSS) a niños y niñas con TEA (Grosberg y Charlop, 2014; Nikopoulos y Keenan, 2004; Wilson, 2013b). Todos han demostrado su eficacia pero, como indican Shukla-Mehta et al. (2010), faltan estudios que ayuden a los investigadores a tomar mejores decisiones a la hora de elegir qué subtipo de VM utilizar, teniendo en cuenta la gran variedad de características comportamentales de los individuos con autismo.

El objetivo de esta revisión es contribuir a una mejor comprensión de los diferentes tipos de VM que existen y realizar un resumen descriptivo y análisis de los resultados de aquellos estudios que han utilizado este método para enseñar habilidades sociales con la finalidad de analizar su eficacia y, siguiendo la línea de algunas investigaciones, saber qué perfil de individuo es el más adecuado para aplicar cada subtipo de VM en la adquisición de HHSS (Corbett y Abdullah, 2005; Wilson, 2013a). También para que en un futuro se puedan establecer guías de intervención de VM y facilitar el trabajo de los investigadores y profesionales la hora de aplicar esta técnica.

Además de la relevancia académica y social del tema objeto de estudio, parece importante señalar que el trabajo en el ámbito de la educación especial con niños con TEA ha hecho patente la importancia de investigar estrategias basadas en la evidencia científica y encaminadas a promover el desarrollo social que nos permitan mejorar la calidad, eficacia y eficiencia de las intervenciones. Ampliar el abanico de propuestas educativas utilizadas para promover el desarrollo cognitivo y social de los niños con TEA tendrá siempre un efecto positivo en el conjunto del sistema educativo y en el futuro de estas personas.

\section{Método}

\subsection{Revisión bibliográfica}

La búsqueda de los trabajos se ha realizado en las bases de datos PsychINFO y ERIC, con la restricción de que sean estudios centrados en VM aplicado a las HHSS y hayan sido publicados en los 15 últimos años. El objetivo es encontrar el mayor número de estudios posibles de este tipo para poder comparar la metodología utilizada, los resultados, categorizarlos en función del tipo de VM que utilizan (Video Self Modeling, peer-VM, adult-VM...) y contribuir al establecimiento de perfiles de individuo para facilitar la aplicación de esta metodología en el futuro. La búsqueda se ha realizado con una combinación de los términos clave: "Autism Spectrum Disorder (ASD)", "Video modeling”, "Social Skills” y "Social Competence”.

\subsection{Criterios de inclusión}

Los criterios de inclusión de los artículos en el presente estudio son:

- Estudios que aporten datos empíricos originales, descartando revisiones sistemáticas y metaanálisis que se han mencionado previamente para repasar la literatura científica. 
- Estudios cuyo objetivo sea aplicar el VM a las HHSS o la competencia social. Este criterio permite descartar todos aquellos estudios que también utilizan VM pero lo aplican a cualquier otra conducta.

- Trabajos que empleen el VM como herramienta principal para la intervención o en combinación con otras técnicas basadas en la evidencia científica.

- Que los participantes de las intervenciones estén diagnosticados de trastorno del espectro autista.

- Trabajos cuyo idioma sea el inglés, porque la búsqueda se hace a nivel internacional, y español.

- Trabajos publicados entre 2002 y 2017.

\subsection{Procedimiento}

La búsqueda se realizó en junio de 2017 y dio lugar en un primer momento a un total de 107 artículos. El primer autor examinó de forma independiente los artículos encontrados en la base de datos PsychINFO y, posteriormente, de forma paralela, el primer y segundo autor examinaron los resúmenes de los artículos encontrados en la base de datos $E R I C$, con el objetivo de seleccionar aquellos que cumplían con los criterios de inclusión. Si los dos autores consideraban que el artículo cumplía los criterios, éste era incluido en la selección. Si no coincidían, el artículo era revisado nuevamente hasta llegar a un consenso. Después de esta primera fase de criba, se rechazaron los artículos duplicados y se seleccionaron un total de 18 artículos. De acuerdo con los criterios de inclusión, y después del acuerdo entre investigadores, se han seleccionado un total de 16 artículos. Todos ellos incluyen el VM como técnica de intervención para fomentar las HHSS en personas con TEA. Finalmente se procedió a extraer la información pertinente de cada uno de ellos.

\subsection{Codificación de los resultados}

Los artículos encontrados han sido categorizados atendiendo a los siguientes parámetros:

- $\quad$ Autor y año de publicación.

- $\quad$ Tipo de diseño utilizado.

- Muestra: número de participantes en cada estudio, edad y contexto en el que se realiza la intervención.

- Principales resultados: haciendo énfasis en los efectos observados y analizando si el resultado es utilizando el VM únicamente o en combinación con otras técnicas.

\section{Resultados}

Los principales resultados obtenidos en cada trabajo pueden observarse en la Tabla 1. A continuación serán descritos según los criterios anteriormente citados. 


\subsection{Participantes}

Los 16 artículos seleccionados incluyen un total de 65 participantes; 59 de ellos son niños con edades que oscilan de los 4 a los 15 años (edad escolar) y diagnóstico de TEA con un nivel de severidad entre moderado y grave. En dos de los artículos 6 participantes son adultos y forman parte de investigaciones que también incluyen a niños a los que se les aplica el VM. En estos dos casos los adultos desempeñan un rol de aprendices y no se consideran parte de la muestra (Acar et al., 2016; Besler y Kurt, 2016).

\subsection{Diseño}

Los 16 artículos seleccionados coinciden en que todos utilizan como diseño de investigación el estudio de caso único y la conducta a aprender está relacionada con la competencia social. Dentro de esta metodología, el diseño de línea base múltiple entre sujetos es el más utilizado: 11 de las investigaciones lo utilizan. Por último, otros 5 estudios utilizan como método el diseño de tratamientos alternantes, que es adecuado para comparar la eficacia de distintos tratamientos en distintos contextos y momentos. En estos estudios como el de Akmanoglu et al. (2014) y Ergenekon et al. (2014), comparan la eficacia del VM versus el modelaje en vivo y también tienen en cuenta si el VM se aplica solo o acompañado de otras técnicas, como pueden ser las incitaciones o el refuerzo.

\begin{tabular}{|c|c|c|c|c|}
\hline Autores & Diseño & $\begin{array}{l}\text { Muestra/ } \\
\text { Participantes }\end{array}$ & Intervención & Resultados \\
\hline $\begin{array}{l}\text { (Acar et al., } \\
\text { 2016) }\end{array}$ & $\begin{array}{l}\text { - Objetivo: enseñar a } \\
\text { las madres de los } \\
\text { participantes a usar VM } \\
\text { e historias sociales y } \\
\text { evaluar la eficacia de } \\
\text { ambos tratamientos. } \\
\text { - Diseño: LBM entre } \\
\text { participantes. } \\
\text { - Tratamientos } \\
\text { alternantes. } \\
\text { - Duración: } 2 \text { meses. }\end{array}$ & $\begin{array}{l}\text { - } \mathrm{N}=3 \text { niños con sus } \\
\text { respectivas madres. } \\
\text { - Edad niños: 6-10 } \\
\text { años. } \\
\text { - Edad madres: 33-45 } \\
\text { años. } \\
\text { - Prerrequisitos } \\
\text { VM: capacidad de } \\
\text { atención de 5', imitar } \\
\text { expresiones verbales, } \\
\text { seguir instrucciones } \\
\text { simples y mirar la } \\
\text { pantalla 2'. }\end{array}$ & $\begin{array}{l}\text { - Contexto: escolar y hogar. } \\
\text { - Tipo VM: Peer VM } \\
\text { individualizado a cada } \\
\text { participante. } \\
\text { - Soporte: ordenador. } \\
\text { - Combinación con otras } \\
\text { técnicas: refuerzo positivo. } \\
\text { - Frecuencia tratamiento: no } \\
\text { especificada. } \\
\text { - Duración VM: no } \\
\text { especificada. }\end{array}$ & $\begin{array}{l}\text { La intervención es eficaz } \\
\text { tanto para entrenar a las } \\
\text { madres a utilizar estas } \\
\text { dos técnicas como para } \\
\text { enseñar a los niños HHSS. } \\
\text { El VM se muestra más } \\
\text { eficiente en dos casos, en } \\
\text { cambio, en el tercer caso, } \\
\text { muestran mayor eficiencia } \\
\text { las historias sociales. } \\
\text { No hay diferencias } \\
\text { significativas entre ambos. }\end{array}$ \\
\hline $\begin{array}{l}\text { (Akmanoglu et } \\
\text { al., 2014) }\end{array}$ & $\begin{array}{l}\text { - Objetivo: enseñar } \\
\text { habilidades de juego } \\
\text { simbólico. } \\
\text { - } \text { Diseño: tratamientos } \\
\text { alternantes. VM y VM } \\
\text { con modelaje en vivo. } \\
\text { - Duración: } 8 \text { sesiones. }\end{array}$ & $\begin{array}{l}\text { - } \mathrm{N}=4 \\
\text { - } \\
\text { - } \text { Prerrequisitos } 4 \text { años. } \\
\text { mismos en el estudio } \\
\text { anterior. }\end{array}$ & $\begin{array}{l}\text { - Contexto: centro } \\
\text { terapéutico. } \\
\text { - Tipo VM: Peer VM, el } \\
\text { mismo vídeo para todos los } \\
\text { participantes. } \\
\text { - Soporte: ordenador. } \\
\text { - Combinación con otras } \\
\text { técnicas: incitaciones. } \\
\text { - Frecuencia: no } \\
\text { especificada. } \\
\text { - Duración VM: no } \\
\text { especificada. }\end{array}$ & $\begin{array}{l}\text { Ambos tratamientos son } \\
\text { efectivos pero la opción de } \\
\text { VM + modelaje en vivo es } \\
\text { ligeramente superior. }\end{array}$ \\
\hline
\end{tabular}




\begin{tabular}{|c|c|c|c|c|}
\hline Autores & Diseño & \begin{tabular}{|l|} 
Muestra/ \\
Participantes \\
\end{tabular} & Intervención & Resultados \\
\hline $\begin{array}{l}\text { (Apple et al., } \\
\text { 2005) }\end{array}$ & $\begin{array}{l}\text { - Objetivo: evaluar la } \\
\text { eficacia del VM con } \\
\text { refuerzo y el VM + } \\
\text { HHSS para aumentar } \\
\text { la frecuencia de las } \\
\text { iniciaciones sociales y } \\
\text { respuestas. } \\
\text { - Diseño: LBM entre } \\
\text { participantes. } \\
\text { - Alternancia de } \\
\text { tratamientos: VM con } \\
\text { refuerzo positivo y VM } \\
\text { con habilidades de } \\
\text { autogestión. } \\
\text { - Duración: no } \\
\text { especificada. } \\
\end{array}$ & 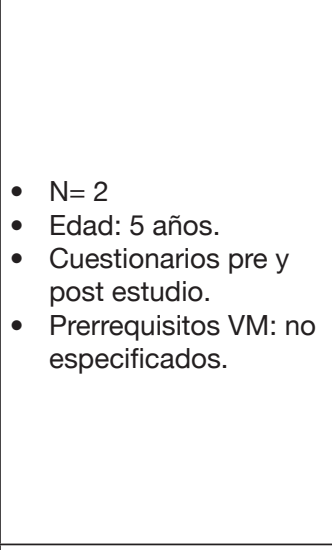 & $\begin{array}{l}\text { - } \text { Contexto: escolar. } \\
\text { compo VM: Peer VM, con } \\
\text { través de cuestionarios } \\
\text { previos. } \\
\text { - Soporte: no especificado. } \\
\text { - Combinación con otras } \\
\text { técnicas: refuerzo } \\
\text { positivo y habilidades de } \\
\text { autogestión. } \\
\text { - Frecuencia: no } \\
\text { especificada. } \\
\text { - Duración VM: no } \\
\text { especificada. }\end{array}$ & $\begin{array}{l}\text { Los resultados muestran } \\
\text { mayor eficacia al combinar } \\
\text { ambos tratamientos (VM y } \\
\text { autogestión) que al utilizar } \\
\text { el VM solo. }\end{array}$ \\
\hline $\begin{array}{l}\text { (Besler y Kurt, } \\
\text { 2016) }\end{array}$ & $\begin{array}{l}\text { - Objetivo: enseñar a } \\
\text { las madres de los } \\
\text { participantes a usar } \\
\text { VM para enseñar } \\
\text { habilidades de juego y } \\
\text { evaluar la eficacia del } \\
\text { tratamiento. } \\
\text { - Diseño: LBM entre } \\
\text { participantes. } \\
\text { - Alternancia de } \\
\text { tratamientos. } \\
\text { Duración: } 1 \text { mes y } \\
\text { medio. }\end{array}$ & $\begin{array}{l}\text { - } \mathrm{N}=6 \\
\text { - } \text { Edad niños: 5-6 años. } \\
\text { - Edad madres: 30-40 } \\
\text { años. } \\
\text { - Prerrequisitos VM: } \\
\text { los mencionados } \\
\text { anteriormente. }\end{array}$ & $\begin{array}{l}\text { - } \text { Contexto: centro } \\
\text { terapéutico privado. } \\
\text { - Tipo de VM: Peer y } \\
\text { Adult VM aplicado } \\
\text { por las madres de los } \\
\text { participantes. } \\
\text { - Soporte: ordenador. } \\
\text { - Combinación con otras } \\
\text { técnicas: refuerzo positivo. } \\
\text { - Frecuencia: } 3 \text { días a la } \\
\text { semana de VM como } \\
\text { mínimo. } \\
\text { - Duración VM: no } \\
\text { especificada. }\end{array}$ & $\begin{array}{l}\text { Los resultados muestran } \\
\text { la efectividad del VM } \\
\text { aplicado por las madres } \\
\text { de los participantes. Éstas } \\
\text { son capaces de aprender } \\
\text { la técnica y administrarla } \\
\text { de forma íntegra. Por } \\
\text { otro lado, todos los } \\
\text { participantes aprenden la } \\
\text { conducta seleccionada. }\end{array}$ \\
\hline $\begin{array}{l}\text { (Boudreau y } \\
\text { Harvey, 2013) }\end{array}$ & $\begin{array}{l}\text { - Objetivo: iniciación } \\
\text { social a un igual. } \\
\text { Diseño: LBM entre } \\
\text { participantes. } \\
\text { - No hay alternancia de } \\
\text { tratamientos. } \\
\text { - Duración: no } \\
\text { especificada. }\end{array}$ & $\begin{array}{l}\text { - } \mathrm{N}=3 \\
\text { - Edad: 4-6 años. } \\
\text { - } \text { Prerrequisitos VM: no } \\
\text { especificados. }\end{array}$ & $\begin{array}{l}\text { - } \text { Contexto: escolar. } \\
\text { Mipo de VM: Video Self } \\
\text { vodeling. Diferentes } \\
\text { cada participante. Dejan } \\
\text { solo comportamientos } \\
\text { adaptativos. } \\
\text { - Soporte: TV. } \\
\text { - Combinación con otras } \\
\text { técnicas: refuerzo positivo. } \\
\text { - Frecuencia VM: no } \\
\text { especificada. } \\
\text { - Duración VM: no } \\
\text { especificada. } \\
\end{array}$ & $\begin{array}{l}\text { Los resultados engrosan } \\
\text { la literatura científica que } \\
\text { afirma que el VSM es una } \\
\text { técnica de aprendizaje } \\
\text { eficaz para niños/as con } \\
\text { TEA. }\end{array}$ \\
\hline $\begin{array}{l}\text { (Ergenekon et } \\
\text { al., 2014) }\end{array}$ & $\begin{array}{l}\text { - Objetivo: enseñar } \\
\text { conductas sociales } \\
\text { complejas (cadena de } \\
\text { acciones). } \\
\text { - Diseño: tratamientos } \\
\text { alternantes.VM y } \\
\text { modelaje en vivo. } \\
\text { - Duración: } 12 \text { sesiones. }\end{array}$ & $\begin{array}{l}\text { - } \quad \mathrm{N}=3 \\
\text { - Edad: 5-10 años. } \\
\text { - Prerrequisitos VM: no } \\
\text { especificados. }\end{array}$ & $\begin{array}{l}\text { - Contexto: hospitalario. } \\
\text { - Tipo de VM: Adult VM } \\
\text { individualizado. } \\
\text { - Soporte: ordenador. } \\
\text { - Combinación con otras } \\
\text { técnicas: refuerzo positivo. } \\
\text { - Frecuencia: } 5 \text { veces/ } \\
\text { - } \text { semana. } \\
\text { - Duración VM: 1-2 min. }\end{array}$ & $\begin{array}{l}\text { No hay diferencias } \\
\text { significativas entre una } \\
\text { intervención u otra. Las } \\
\text { dos son efectivas. } \\
\text { Resultados contradictorios } \\
\text { en cuanto a eficiencia. }\end{array}$ \\
\hline
\end{tabular}




\begin{tabular}{|c|c|c|c|c|}
\hline Autores & Diseño & $\begin{array}{l}\text { Muestra/ } \\
\text { Participantes }\end{array}$ & Intervención & Resultados \\
\hline $\begin{array}{l}\text { (Macpherson et } \\
\text { al., 2015) }\end{array}$ & $\begin{array}{l}\text { - Objetivo: enseñar } \\
\text { variedad de respuestas } \\
\text { y comentarios sociales } \\
\text { (cumplidos y gestos) } \\
\text { durante una actividad. } \\
\text { - Diseño: LBM entre } \\
\text { participantes. } \\
\text { - No hay alternancia de } \\
\text { tratamientos. } \\
\text { Duración: } 1 \text { mes y } \\
\text { medio. }\end{array}$ & $\begin{array}{ll}\text { - } & \mathrm{N}=5 \\
\text { - } & \text { Edad: 9-11 años. } \\
\text { - } & \text { Prerrequisitos VM: no } \\
& \text { especificados. }\end{array}$ & $\begin{array}{l}\text { - } \text { Contexto: parque. } \\
\text { - Vídeos de VM: Adult VM. } \\
\text { - Soporte: Ipad. } \\
\text { - Combinación con otras } \\
\text { técnicas: no especificado. } \\
\text { - Frecuencia: no } \\
\text { especificada. } \\
\text { - Duración VM: } 20 " .\end{array}$ & $\begin{array}{l}\text { Éxito: participante } \\
\text { responde bien antes de } \\
5 \text { intentos. Todos los } \\
\text { participantes mejoran } \\
\text { sus respuestas aunque la } \\
\text { generalización es limitada }\end{array}$ \\
\hline $\begin{array}{l}\text { (Charlop et al., } \\
\text { 2010) }\end{array}$ & $\begin{array}{l}\text { Objetivo: hacer } \\
\text { comentarios. } \\
\text { adecuados, gestos y } \\
\text { expresiones faciales } \\
\text { dentro de una } \\
\text { conversación. } \\
\text { Diseño: LBM entre } \\
\text { participantes. } \\
\text { No hay alternancia de } \\
\text { tratamientos. } \\
\text { Duración: No } \\
\text { especificada. }\end{array}$ & $\begin{array}{ll}\text { - } & \mathrm{N}=3 \\
\text { - } & \text { Edad: } 7-11 \text { años. } \\
\text { - } & \text { Prerrequisitos VM: no } \\
& \text { especificados. }\end{array}$ & $\begin{array}{l}\text { - } \text { Contexto: centro } \\
\text { terapéutico privado. } \\
\text { - Tipo de VM: Adult VM } \\
\text { individualizado. } \\
\text { - } \text { Soporte: TV. } \\
\text { - Combinación con otras } \\
\text { técnicas: no especificado. } \\
\text { - Frecuencia: no } \\
\text { especificada. } \\
\text { Duración VM: no } \\
\text { especificada. }\end{array}$ & $\begin{array}{l}\text { El VM se muestra eficaz } \\
\text { para enseñar este tipo de } \\
\text { conductas. }\end{array}$ \\
\hline $\begin{array}{l}\text { (Miltenberger y } \\
\text { Charlop, 2015) }\end{array}$ & $\begin{array}{l}\text { Objetivo: aumento de } \\
\text { comentarios verbales, } \\
\text { juego social y aumento } \\
\text { de demandas. } \\
\text { - Diseño: LBM entre } \\
\text { participantes. } \\
\text { - No hay alternancia de } \\
\text { tratamientos. } \\
\text { - Duración: 4-6 semanas. }\end{array}$ & $\begin{array}{ll}\text { - } & \mathrm{N}=5 \\
\text { - } & \text { Edad: 5-12 años. } \\
\text { - } & \text { Prerrequisitos VM: no } \\
& \text { especificados. }\end{array}$ & $\begin{array}{l}\text { - Contexto: centro de } \\
\text { terapia. } \\
\text { - Tipo de VM: Adult VM (TV) } \\
\text { vs. VM en ipad. Vídeos } \\
\text { individualizados. } \\
\text { - Soporte: TV y Ipad. } \\
\text { - Combinación con otras } \\
\text { técnicas: no especificado. } \\
\text { - Frecuencia: no } \\
\text { especificada. } \\
\text { - Duración: } 30 "-112 " . \\
\end{array}$ & $\begin{array}{l}\text { Los dos tipos de VM } \\
\text { son efectivos, aunque } \\
\text { los resultados no son } \\
\text { concluyentes. }\end{array}$ \\
\hline $\begin{array}{l}\text { (Nikopoulos y } \\
\text { Keenan, 2004) }\end{array}$ & $\begin{array}{l}\text { - Objetivo: aumento de } \\
\text { iniciaciones sociales y } \\
\text { juego recíproco. } \\
\text { - Diseño: LBM entre } \\
\text { participantes. } \\
\text { - No hay alternancia de } \\
\text { tratamientos. } \\
\text { - Duración: 3-4 semanas } \\
\text { + seguimiento. }\end{array}$ & $\begin{array}{ll}\text { - } & \mathrm{N}=3 \\
\text { - } & \text { Edad: 7-9 años. } \\
\text { - } & \text { Prerrequisitos VM: no } \\
& \text { especificados. }\end{array}$ & $\begin{array}{l}\text { - Contexto: centro } \\
\text { terapéutico. } \\
\text { - Tipo de VM: Peer VM. } \\
\text { - Soporte: TV. } \\
\text { - Combinación con otras } \\
\text { técnicas: no especificado. } \\
\text { - Frecuencia: no } \\
\text { especificada. } \\
\text { - Duración: } 35 " .\end{array}$ & $\begin{array}{l}\text { El Peer VM se muestra } \\
\text { eficaz para aumentar las } \\
\text { iniciaciones sociales y el } \\
\text { juego recíproco. }\end{array}$ \\
\hline
\end{tabular}




\begin{tabular}{|c|c|c|c|c|}
\hline Autores & Diseño & \begin{tabular}{|l|} 
Muestra/ \\
Participantes
\end{tabular} & Intervención & Resultados \\
\hline $\begin{array}{l}\text { (Nikopoulos y } \\
\text { Keenan, 2003) }\end{array}$ & $\begin{array}{l}\text { - Objetivo: promover las } \\
\text { iniciaciones sociales en } \\
\text { un contexto de juego. } \\
\text { - Diseño: LBM entre } \\
\text { participantes. } \\
\text { - No hay alternancia de } \\
\text { tratamientos. } \\
\text { - Duración: No } \\
\text { especificada. }\end{array}$ & $\begin{array}{ll}- & \mathrm{N}=7 \\
\text { - } & \text { Edad: 9-15 años. } \\
\text { - } & \text { Prerrequisitos VM: no } \\
& \text { especificados. }\end{array}$ & $\begin{array}{l}\text { - } \text { Contexto: escuela de } \\
\text { educación especial. } \\
\text { - Tipo de VM: Peer VM y } \\
\text { Adult VM (con familiares y } \\
\text { desconocidos). } \\
\text { - Soporte: TV. } \\
\text { - Combinación con otras } \\
\text { técnicas: no especificado. } \\
\text { - Frecuencia: no } \\
\text { - } \text { especificada. } \\
\text { - Duración VM: } 35 " .\end{array}$ & $\begin{array}{l}\text { Los diferentes tipos de } \\
\text { VM (Peer y Adult) son } \\
\text { efectivos para aumentar } \\
\text { las iniciaciones sociales en } \\
4 \text { de los } 7 \text { participantes. } \\
\text { Los otros } 3 \text { no mostraron } \\
\text { mejora probablemente } \\
\text { debido a la presencia } \\
\text { de conducta disruptiva } \\
\text { durante las sesiones y la } \\
\text { ausencia de habilidades de } \\
\text { juego. }\end{array}$ \\
\hline $\begin{array}{l}\text { (Sani-Bozkurt y } \\
\text { Ozen, 2015) }\end{array}$ & $\begin{array}{l}\text { - Objetivo: enseñar } \\
\text { habilidades de juego } \\
\text { simbólico. } \\
\text { - Diseño: tratamientos } \\
\text { alternantes. Peer-VM y } \\
\text { Adult VM. } \\
\text { - Duración: 8-12 } \\
\text { sesiones. }\end{array}$ & $\begin{array}{l}\text { - } \quad \mathrm{N}=3 \\
\text { - } \\
\text { - } \\
\text { Pdad: 5-6 añosuisitos VM: no } \\
\text { especificados. }\end{array}$ & $\begin{array}{l}\text { - } \text { Contexto: centro } \\
\text { terapéutico. } \\
\text { - Tipo de VM: Peer VM. EI } \\
\text { mismo vídeo para todos los } \\
\text { participantes. } \\
\text { - Soporte: ordenador. } \\
\text { - Combinación con otras } \\
\text { técnicas: no especificado. } \\
\text { - Frecuencia: no } \\
\text { especificada. } \\
\text { - Duración VM: no } \\
\text { especificada. }\end{array}$ & $\begin{array}{l}\text { No existen diferencias } \\
\text { significativas entre usar } \\
\text { un adulto como modelo o } \\
\text { un igual. Ambos tipos son } \\
\text { efectivos para enseñar } \\
\text { habilidades de juego } \\
\text { simbólico a niños con TEA. }\end{array}$ \\
\hline $\begin{array}{l}\text { (Ulke- } \\
\text { Kurkcuoglu, } \\
\text { 2015) }\end{array}$ & $\begin{array}{l}\text { - Objetivo: enseñar } \\
\text { habilidades } \\
\text { relacionadas con el } \\
\text { juego social. } \\
\text { - Diseño: tratamientos } \\
\text { alternantes.VM e } \\
\text { incitaciones (de menos } \\
\text { a más). } \\
\text { - Duración: 1-2 meses. }\end{array}$ & $\begin{array}{l}\text { - } \mathrm{N}=3 \\
\text { - Edad: 5-6 años. } \\
\text { - Prerrequisitos } \\
\text { VM: capacidad de } \\
\text { atención de 5', imitar } \\
\text { expresiones verbales, } \\
\text { seguir instrucciones } \\
\text { simples y mirar la } \\
\text { pantalla 2'. }\end{array}$ & $\begin{array}{l}\text { - Contexto: no especificado. } \\
\text { - Tipo de VM: Peer VM. } \\
\text { Vídeos individualizados. } \\
\text { - Soporte: ordenador. } \\
\text { - } \text { técnbinación con otras } \\
\text { - Frecuencia: no } \\
\text { especificada. } \\
\text { - Duración VM: 1'20". }\end{array}$ & $\begin{array}{l}\text { Comparan resultados de } \\
\text { ambos tratamientos: las } \\
\text { dos técnicas son igual } \\
\text { de efectivas pero es más } \\
\text { eficiente la incitación que } \\
\text { el VM. }\end{array}$ \\
\hline $\begin{array}{l}\text { (Wert y } \\
\text { Neisworth, } \\
\text { 2003) }\end{array}$ & $\begin{array}{l}\text { - Objetivo: comprobar la } \\
\text { eficacia del VSM para } \\
\text { aumentar la frecuencia } \\
\text { de preguntas } \\
\text { espontáneas. } \\
\text { - Diseño: LBM entre } \\
\text { participantes. } \\
\text { - No hay alternancia de } \\
\text { tratamientos. } \\
\text { - Duración: No } \\
\text { especificada. }\end{array}$ & $\begin{array}{l}\text { - } \mathrm{N}=4 \\
\text { - } \quad \text { Edad: } 3-6 \text { años. } \\
\text { - Prerrequisitos VM: no } \\
\text { especificados. }\end{array}$ & $\begin{array}{l}\text { - Contexto: centro } \\
\text { terapéutico y hogar familiar. } \\
\text { - Tipo de VM: VSM. Vídeos } \\
\text { individualizados } \\
\text { - Soporte: TV. } \\
\text { - Combinación con otras } \\
\text { técnicas: refuerzo positivo. } \\
\text { - Frecuencia: no } \\
\text { especificada. } \\
\text { - Duración VM: } 5 \text {. }\end{array}$ & $\begin{array}{l}\text { EI VSM se muestra } \\
\text { efectivo. Todos los } \\
\text { participantes aumentan la } \\
\text { frecuencia de preguntas } \\
\text { espontáneas aunque uno } \\
\text { de ellos de forma menos } \\
\text { pronunciada debido a sus } \\
\text { características. }\end{array}$ \\
\hline $\begin{array}{l}\text { (Williamson et } \\
\text { al., 2012) }\end{array}$ & $\begin{array}{l}\text { - Objetivo: saludar por } \\
\text { iniciativa propia. } \\
\text { - Diseño: LBM entre } \\
\text { participantes. } \\
\text { - No hay alternancia de } \\
\text { tratamientos. } \\
\text { - Duración: } 1-2 \text { meses. }\end{array}$ & $\begin{array}{l}\text { - } \quad \mathrm{N}=3 \\
\text { - Edad: 7-10 años. } \\
\text { - Prerrequisitos VM: } \\
\text { los mismos que } \\
\text { en los estudios ya } \\
\text { mencionados. }\end{array}$ & $\begin{array}{l}\text { - Contexto: escolar. } \\
\text { - Tipo de VM: VSM. } \\
\text { - Soporte: Ipod. } \\
\text { - } \text { Combinación con otras } \\
\text { técnicas: no especificado. } \\
\text { - Frecuencia: no } \\
\text { - } \quad \text { Duracificad: } 30 "-1\end{array}$ & $\begin{array}{l}\text { EI VSM solo se muestra } \\
\text { eficaz en un caso. En } \\
\text { los otros dos no se } \\
\text { observa aprendizaje de la } \\
\text { conducta. Los resultados } \\
\text { correlacionan con el nivel } \\
\text { de habilidades previas } \\
\text { de cada niño: solo uno } \\
\text { de ellos tenía buenas } \\
\text { puntuaciones. }\end{array}$ \\
\hline
\end{tabular}




\begin{tabular}{|c|c|c|c|c|}
\hline Autores & Diseño & $\begin{array}{l}\text { Muestra/ } \\
\text { Participantes }\end{array}$ & Intervención & Resultados \\
\hline (Wilson, 2013a) & $\begin{array}{l}\text { - Objetivo: potenciar la } \\
\text { comunicación social. } \\
\text { Diseño: tratamientos } \\
\text { alternantes. VM con } \\
\text { modelaje en vivo. } \\
\text { - Duración: 1-2 meses. }\end{array}$ & $\begin{array}{ll}- & \mathrm{N}=5 \\
\text { - } & \text { Edad preescolar. } \\
\text { - } & \text { Prerrequisitos: los } \\
& \text { mismos que en el } \\
& \text { estudio de Ulke- } \\
& \text { Kurkuoglu (2015). }\end{array}$ & $\begin{array}{l}\text { - } \text { Contexto: escolar. } \\
\text { - } \text { Profeso de VM: Adult VM. } \\
\text { - Soporte: no especificado. } \\
\text { - Combinación con otras } \\
\text { técnicas: no especificado. } \\
\text { - Frecuencia: } 3 \text { veces/ } \\
\text { semana. } \\
\text { - Duración: 3'. }\end{array}$ & $\begin{array}{l}\text { Las dos intervenciones son } \\
\text { eficaces pero sobresale } \\
\text { más el VM. } \\
\text { Conclusión: en función del } \\
\text { tipo de alumno se puede } \\
\text { beneficiar más de un tipo } \\
\text { de técnica u otra. }\end{array}$ \\
\hline
\end{tabular}

Fuente: elaboración propia.

\subsection{Tipo de VM}

Respecto al tipo de VM utilizado, 8 de las investigaciones utilizan el Peer-VM (con iguales) alternando tratamientos con otras estrategias como el modelaje en vivo y en dos casos, lo combinan con Adult-VM para comparar los resultados entre ambos (Nikopoulos y Keenan, 2003; Sani-Bozkurt y Ozen, 2015). Tres de los estudios se centran en aplicar VSM combinado con otras estrategias como el refuerzo positivo con el objetivo de potenciar los resultados. Respecto al Adult VM, 5 estudios lo utilizan como técnica principal ya sea como única técnica o alternando tratamientos como el modelaje en vivo. Finalmente, hay 1 estudio que no se puede clasificar en función del tipo de VM porque utiliza las historias sociales de Gray en formato vídeo como modelo para enseñar habilidades sociales a los participantes (Halle et al., 2016).

El estudio de Sani-Bozkurt y Ozen (2015) indica que no existen diferencias significativas en la utilización de modelos adultos o iguales. No se ha encontrado ningún estudio reciente que compare el VSM con los otros dos tipos de VM (adultos o iguales) en el ámbito de las HHSS, pero sí se encuentran en la literatura científica otros estudios, centrados en conductas más simples, que comparan los diferentes tipos de VM.

Finalmente, tal como muestran los resultados relativos al soporte utilizado para aplicar el VM, estos indican que no existen diferencias significativas entre los diferentes soportes existentes, ya sea TV, tablet o bien ordenador.

\subsection{Contexto}

Respecto al contexto donde se realizan las intervenciones, la mitad de estas han sido llevadas a cabo en el contexto hospitalario o centro terapéutico y la mitad restante en contextos naturales como son la escuela, el hogar familiar o el parque. Todos han obtenido resultados positivos en cuanto a eficacia. 


\section{Eficacia, eficiencia y generalización de los resultados}

Los resultados muestran que el VM es un método eficaz para enseñar HHSS a niños y niñas con TEA. Solo en dos de las investigaciones que comparan el VM con el modelaje en vivo (Ergenekon et al., 2014) y las incitaciones (Ulke-Kurkcuoglu, 2015) podemos observar que los resultados respecto la eficiencia varían en función de cada participante; en el primer caso no se pueden obtener conclusiones generales y, en el segundo, observamos mayor eficiencia de las incitaciones respecto al VM. Pero hay que tener en cuenta, como hemos visto en otros estudios (Wilson, 2013b), la importancia de que los participantes cumplan unos requisitos previos a la aplicación del VM como capacidad de atención sostenida de 5 minutos, capacidad de imitar expresiones verbales, seguir instrucciones simples y capacidad de mirar una pantalla durante al menos 2 minutos. En la intervención de Wilson (2013b), que también compara la eficacia de estas dos técnicas y tiene en cuenta los requisitos previos, observamos que el VM obtiene mayor eficacia y eficiencia que el modelaje en vivo. En otras investigaciones que comparan tratamientos como la de Akmanoglu et al. (2014) y Apple et al. (2005) podemos observar que la combinación de VM con otras técnicas como las incitaciones, el modelaje en vivo y las autoinstrucciones resulta más eficaz que el VM solo.

Nueve de los estudios utilizan el VM como técnica principal de intervención, que puede ir acompañada o no de otras técnicas como el refuerzo positivo o las recompensas. Los vídeos utilizados están individualizados en función de las necesidades de cada participante (Macpherson et al., 2015; Charlop et al., 2010; Williamson et al., 2012) y todos obtienen resultados positivos al respecto.

En relación a la generalización de las conductas, la mayoría de las investigaciones (Acar et al., 2016; Akmanoglu et al., 2014; Besler y Kurt, 2016; Charlop et al., 2010; Ergenekon et al., 2014; Macpherson et al., 2015; Miltenberger y Charlop, 2015; Nikopoulos y Keenan, 2003; Sani-Bozkurt y Ozen, 2015; Ulke-Kurkcuoglu, 2015), incluyen una fase para medir los efectos del mantenimiento y generalización de los aprendizajes; aspecto que tiene mucho interés teórico y práctico. Los estudios seleccionados han medido la generalización observando la conducta objetivo en diferentes contextos, actividades o personas antes y después de la intervención con la finalidad de comparar los resultados y estudiar si la conducta enseñada se ha consolidado. El uso de instrucciones verbales y refuerzos en algunos de los estudios parece ser más efectivo para promover la adquisición, el mantenimiento y la generalización de la conducta (Miltenberger y Charlop, 2015). Por último, de todos los estudios seleccionados, cuatro de ellos (Apple et al., 2005; Boudreau y Harvey, 2013; Nikopoulos y Keenan, 2004; Wert y Neisworth, 2003) no evalúan la generalización y, en dos de ellos (Williamson et al., 2012; Wilson, 2013b), a pesar de haber tomado medidas al respecto, no analizan los datos en los resultados o estos no son suficientemente específicos y, por lo tanto, no se pueden extraer conclusiones al respecto. 


\section{Discusión}

Si comparamos la efectividad del VM con el modelaje in vivo los resultados indican que ambas estrategias se muestran efectivas, en ocasiones siendo el VM incluso más efectivo que el modelaje in vivo (CharlopChristy et al., 2000); sin embargo, Wilson (2013b) afirma que no siempre es así. En su estudio, por un lado, observa que los patrones de atención visual de los alumnos son mayores durante la aplicación del VM que durante el modelaje in vivo, por ello, destaca el uso de las nuevas tecnologías en intervenciones con personas con TEA por su potencial para captar su atención. Por otro lado, con dos de los participantes es el modelaje in vivo el que obtiene mejores resultados. Lo mismo ocurre con los estudios realizados por Akmanoglu et al. (2014) y Ergenekon et al. (2014), que comparan la eficacia del VM solo o combinado con modelaje in vivo: las dos técnicas son eficaces pero, con uno de los participantes en cada estudio, observan que el VM obtiene peores resultados que utilizado de forma combinada. Para explicar esta diferencia, Wilson (2013b), Akmanoglu (2015) y Ergenekon et al. (2014) coinciden en señalar la existencia de otras variables que influyen en la efectividad del VM y pueden hacer variar los resultados, como pueden ser: la complejidad del diagnóstico de TEA (comorbilidades), que puede aumentar la falta de respuesta al tratamiento; la conducta y contextos seleccionados para el individuo en concreto, que pueden tener menor valor reforzante en función de su naturaleza; y por último, el hecho de que existe un subgrupo de individuos con TEA con los que el VM no se muestra efectivo. En consecuencia, antes de aplicar VM nos debemos asegurar de que los participantes cumplan unos requisitos previos: capacidad de atención sostenida de 5 minutos, capacidad de imitar expresiones verbales, seguir instrucciones simples de dos o tres órdenes; en caso contrario, es mejor utilizar el modelaje in vivo. Además de tener en cuenta el valor reforzante y la complejidad de la conducta seleccionada para cada individuo.

Otra posible explicación de este hecho sería la propuesta hecha por Wilson (2013b), donde apunta que el nivel de habilidades previas de cada individuo está directamente relacionado con la mayor o menor eficacia del VM.

Los datos analizados muestran que la edad no es un factor determinante en la intervención con VM. Respecto a la generalización del aprendizaje, en el caso de individuos con dificultades de comunicación social y/o discapacidad intelectual, es preferible aplicar las intervenciones en su comunidad, ya sea el hogar o la escuela, para facilitar la consolidación del mismo (Odom et al., 2008).

El análisis del VM como herramienta para enseñar HHSS a personas con TEA muestra resultados positivos, excepto el estudio de Williamson et al. (2012) que explica que el motivo por el que el VM no ha resultado eficaz en todos los participantes está relacionado con el nivel previo de habilidades de cada uno. Como se ha comentado anteriormente, para la aplicación de esta técnica es importante que los niños y niñas cumplan unos criterios previos y, como aclara el autor en su estudio, solo uno de los participantes los cumplía todos; el resto solo cumplía algunos de ellos y es precisamente en esos casos en los que el VM no ha mostrado efecto.

Estos puntos en común dirigen las futuras investigaciones en este ámbito y sirven para establecer orientaciones para poner en práctica esta técnica. Los resultados muestran algunas características del perfil de sujeto adecuado para aplicar VM como son: (1) Las conductas elegidas deben estar adaptadas a cada 
individuo en particular en función de sus necesidades y características (Shukla-Mehta et al., 2010; Wilson, 2013a); (2) Cada participante debe cumplir unos prerrequisitos mínimos que garanticen la capacidad de: mantener la atención el tiempo necesario para ver el vídeo, disponer de habilidades básicas de imitación (verbal y motora), capacidad de procesamiento de la información visual y presentar habilidades de juego funcional. Esta última característica está relacionada con el concepto de zona de desarrollo próximo (Vigotsky, 1978); (3) Que los vídeos tengan una duración corta y la conducta objetivo se muestre dividida en unidades más pequeñas con el fin de facilitar la comprensión de lo que deben aprender (Nikopoulos y Nikopoulou-Smyrni, 2008) y, por último, (4) elegir conductas que se puedan observar fácilmente; pues no todas las conductas son susceptibles de utilizar con VM (Wert y Neisworth, 2003; Wilson, 2013a). Parece pues necesario evaluar las habilidades de cada niño en atención sostenida, imitación, procesamiento visual y seguimiento de instrucciones complejas antes de realizar la intervención (Akmanoglu et al., 2014; UlkeKurkcuoglu, 2015; Williamson et al., 2012; Wilson, 2013b) con la finalidad de que el VM sea efectivo. En definitiva, puede concluirse que cuanto mayores sean la habilidades previas del niño, mejores resultados obtendrá al finalizar la intervención (Williamson et al., 2012)

En todos los estudios, los participantes están en edad escolar y tienen un nivel de afectación moderado (según criterios del DSM 5). También hay que valorar el tipo de conducta que se quiere enseñar; los estudios analizados en esta revisión coinciden en que las conductas elegidas se caracterizan por ser fácilmente observables y cuantificables (expresiones verbales, acciones, gestos...) y han sido elegidas a partir de la información obtenida a través de entrevistas con los familiares y educadores y también de la observación directa de los participantes.

Las personas con TEA se caracterizan por presentar alteraciones de la comunicación social, que surgen de la interacción. Esta alteración afecta no solamente a la comprensión y expresión del lenguaje, sino a la capacidad de simbolizar en general (formar y relacionar conceptos, resolver problemas, mantener la atención, anticipar...) (Rivière y Núñez, 2008). La función simbólica es la capacidad de imaginar y recordar situaciones, objetos, animales o acciones sin que estén presentes en ese momento ni sean percibidas por los sentidos (Ruiz de Velasco y Abad, 2011). Para Vigotsky (1978), la comunicación está en el origen del pensamiento y afirma que no puede haber desarrollo psicológico sin la participación de otras personas. Según la manera en la que se va tramando la comunicación, dependerá la posibilidad de construir posteriormente sistemas simbólicos más complejos como el lenguaje verbal (Ruiz de Velasco y Abad, 2011). Atendiendo a todas estas dificultades y conociendo la gran variedad de perfiles de personas con TEA, es importante encontrar diferentes métodos educativos, basados en la evidencia científica, que sirvan para enseñar distintos tipos de conductas; además, éstos deben adaptarse a las características de cada caso particular. En relación a la variabilidad de las personas con TEA, parece importante que se siga engrosando la literatura científica en este ámbito, con diferentes subtipos de conducta social y diferentes modelos (profesores, padres de los participantes...) y contextos (escuela, hogar de los participantes...) con el fin de ampliar los recursos existentes y que éstos se muestren eficaces para los diferentes tipos de TEA.

Como se ha comentado en el apartado de resultados, algunos de los estudios señalan como reto pendiente mejorar la generalización de los aprendizajes durante las intervenciones. Una manera de hacerlo, como señala Wilson (2013b) es utilizando diseños de línea base múltiple, y en aquellos casos en que se utilice el diseño de tratamientos alternantes, también se puede establecer una línea base, aunque por el tipo de diseño no sea obligatorio. Siguiendo con la misma autora, también se pueden realizar estudios de seguimiento una 
vez realizada la intervención, y medir el mantenimiento y la generalización de la conducta pasado un tiempo. Los artículos más recientes tienen en cuenta este aspecto y la revisión realizada resume los puntos indicados por algunos de los estudios de la Tabla 1 y proporciona pautas para futuras investigaciones y/o profesionales que quieran utilizar el VM (Acar et al., 2016; Apple et al., 2005; Nikopoulos y Keenan, 2004; Williamson et al., 2012). Los aspectos que deberían tenerse en cuenta son: 1) La utilización de diferentes vídeos durante las sesiones de entrenamiento, por ejemplo, pueden enseñar vídeos de la conducta a aprender en diferentes contextos y con personas distintas pero manteniendo la misma conducta, como un saludo o un gesto particular; 2) La evaluación de la conducta a enseñar pre-intervención en distintos contextos y con distintas personas y evaluación post-intervención de la misma conducta en las mismas circunstancias para poder comparar los resultados; 3) Una vez asumidos los criterios de dominio de la conducta, la eliminación paulatina del refuerzo o las incitaciones dadas al participante para promoverla. Estos puntos son de especial relevancia teniendo en cuenta las dificultades de las personas con TEA para generalizar los aprendizajes y porque, como señalan Baer et al. (1968), se entiende que un individuo ha generalizado su conducta cuando ésta aparece en una variedad de contextos o si ésta permanece a lo largo del tiempo. Ambos criterios son objetivos de todos los estudios analizados. Se necesitan más estudios que utilicen vídeos en diferentes contextos para fomentar la generalización y que hagan seguimiento durante más tiempo.

Algunas limitaciones de los estudios son que en las muestras de participantes no hay casos de TEA graves. Por tanto, las futuras investigaciones deberían centrarse en incluir individuos con distintos tipos y niveles de intensidad de apoyo con el fin de poder comparar los resultados entre ellos y ampliar las posibilidades de intervención en aquellos casos de autismo más graves. Por otro lado, estos estudios difieren en el tipo de VM utilizado y en que la mayoría de ellos han aplicado el VM conjuntamente a otras técnicas como el refuerzo positivo, incitaciones, premios, etc. En consecuencia, se hace difícil distinguir si el resultado del estudio se debe únicamente al VM o a otras variables que también han contribuido al aprendizaje del niño. En algunos estudios (Macpherson et al., 2015) el hecho de añadir refuerzo y feedback durante la intervención ha sido necesario para mejorar la frecuencia de la conducta a aprender. Respecto al tipo de VM a utilizar, no hay diferencias significativas en los resultados dado que todos se muestran igual de efectivos. Aunque como señala Wilson (2013a), parece más adecuado utilizar peer VM cuando se trata de enseñar HHSS para que el participante pueda ponerlo en práctica con un compañero que le resulte familiar. Faltan investigaciones en este sentido. Por este motivo, es importante que se siga investigando en este campo, para medir y distinguir el efecto específico del VM en relación con otras técnicas como el refuerzo y facilitar futuras intervenciones que puedan elegir qué tipo de VM es más adecuado teniendo en cuenta las diferencias individuales en las habilidades del alumnado con TEA. Por ahora, la mejor manera de elegir qué tipo de VM utilizar parece ser seguir los requisitos mencionados anteriormente, valorando las características de la persona, la conducta objetivo y el contexto en el que se realizará. 


\section{Referencias bibliográficas}

Acar, C. et al. (2016): "Effects of Mother-Delivered Social Stories and Video Modeling in Teaching Social Skills to Children With Autism Spectrum Disorders". The Journal of Special Education, 50 (4): 215-226.

Akmanoglu, N. (2015): "Effectiveness of teaching naming facial expression to children with autism via video modeling". Kuram ve Uygulamada Egitim Bilimleri, 15 (2).

Akmanoglu, N. et al. (2014): "Comparing Video Modeling and Graduated Guidance Together and Video Modeling Alone for Teaching Role Playing Skills to Children with Autism". Education and Training in Autism and Developmental Disabilities, 49 (1): 17-31.

American Psychiatric Association (2013): Diagnostic and statistical manual of mental disorders. 5th ed. Washington: American Psychiatric Association.

Apple, A. et al. (2005): "Effects of Video Modeling Alone and With Self-Management on Compliment-Giving Behaviors of Children with High-Functioning ASD". Journal of Positive Behavior Interventions, 7 (1), 33-46.

Baer, D. M. et al. (1968): "Some current dimensions of applied behavior analysis". Journal of Applied Behavior Analysis, 1 (1), 91-97.

Baron-Cohen, S. et al. (1985): "Does the autistic child have a "theory of mind"?*”. Cognition, 21: 37-46.

Bellini, S. y Akullian, J. (2007): "A Meta-Analysis of Video Modeling and Video Self-Modeling Interventions for Children and Adolescents with Autism Spectrum Disorders". Exceptional Children, 73 (3), 264-287.

Besler, F. y Kurt, O. (2016): "Effectiveness of video modeling provided by mothers in teaching play skills to children with autism". Kuram ve Uygulamada Egitim Bilimleri, 16 (1): 209-230.

Boudreau, J. y Harvey, M. (2013): “Increasing Recreational Initiations for Children Who Have ASD Using Video Self Modeling". Education \& Treatment of Children, 36 (1): 49-60.

Charlop-Christy, M. H. et al. (2010): "Teaching Socially Expressive Behaviors to Children with Autism Through Video Modeling". Education and Treatment of Children, 33 (3), 371-393.

Charlop-Christy, M. H. et al. (2000): "A comparison of video modeling with in vivo modeling for teaching children with autism". Journal of Autism and Developmental Disorders, 30 (6): 537-552.

Christensen, D. et al. (2016): "Prevalence and Characteristics of Autism Spectrum Disorder Among Children Aged 8 Years - Autism and Developmental Disabilities Monitoring Network, 11 Sites, United States, 2012”. Morbidity and mortality weekly report. Surveillance summaries, 65 (3): 1-23 (en línea). <http://www.cdc.gov/mmwr/volumes/65/ ss/ss6503a1.htm\%5Cnhttp://www.ncbi.nlm.nih.gov/pubmed/27031587>, acceso 29 de mayo de 2018.

Corbett, B. A. (2003): "Video Modeling : A window into the world of autism". The Behavior Analyst Today, 4 (3): 367-377.

Corbett, B. A. y Abdullah, M. (2005): "Video Modeling: Why does it work for children with autism?". Journal of Early and Intensive Behavior Intervention, 2 (1): 2-8.

Delano, M. (2007): "Video Modeling Interventions for Individuals with Autism”. Remedial and Special Education, 28 (1), 33-42. 
Ergenekon, Y. et al. (2014): "Comparison of video and live modeling in teaching response chains to children with autism". Education in Training in Autism and Developmental Disabilities, 49 (2): 200-213.

Grosberg, D. y Charlop, M. (2014): "Teaching Persistence in Social Initiation Bids to Children with Autism Through a Portable Video Modeling Intervention (PVMI)". Journal of Developmental and Physical Disabilities, 26 (5): 527-541.

Halle, S. et al. (2016): "Teaching social skills to students with autism: a video modeling social stories approach". Behavior and Social Issues, 25: 42-63.

Koegel, R. et al. (2014): "Improving question-asking initiations in young children with autism using pivotal response treatment". Journal of Autism and Developmental Disorders, 44 (4), 816-827.

Macpherson, K. et al. (2015): "Using Portable Video Modeling Technology to Increase the Compliment Behaviors of Children with Autism During Athletic Group Play". Journal of Autism and Developmental Disorders, 45 (12): 3836-3845.

Miltenberger, C., y Charlop, M. (2015): “The Comparative Effectiveness of Portable Video Modeling vs. Traditional Video Modeling Interventions with Children with Autism Spectrum Disorders”. Journal of Developmental and Physical Disabilities, 27 (3): 341-358.

Newton, E., y Jenvey, V. (2011): "Play and theory of mind: associations with social competence in young children". Early Child Development and Care, 181 (6): 761-773.

Nikopoulos, C., y Keenan, M. (2003): "Promoting social initiation in children with autism using video modeling". Behavioral Interventions, 18 (2): 87-108.

Nikopoulos, C., y Keenan, M. (2004): "Effects of video modeling on social initiations by children with autism". Journal of Applied Behavior Analysis, 37 (1): 93-96.

Nikopoulos, C., y Nikopoulou-Smyrni, P. (2008): "Teaching Complex Social Skills To Children With Autism; Advances of Video Modeling". Journal of Early and Intensive Behavior Intervention, 5 (2): 30-43.

Odom, S. et al. (2005): "Research in special education: Scientific methods and evidence-based practices". Exceptional Children, 71 (2): 137-148.

Odom, S. et al. (2008): "Social competence of young children: conceptualization, assesment, and influences", en Brown, W. et al. (eds.): Social competence of young children: Risk, Disability, and Intervention. Baltimore: Paul H. Brookes Pub.

Odom, S. et al. (2006): "Social acceptance and rejection of preschool children with disabilities: A mixed-method analysis". Journal of Educational Psychology, 98 (4): 807-823.

Ozonoff, S. y Miller, J. (1995): "Teaching theory of mind: a new approach to social skills training for individuals with autism". Journal of Autism and Developmental Disorders, 25: 415-433.

Perinat, A. (2007): Psicología del desarrollo: un enfoque sistémico. Barcelona: UOC.

Reichow, B. y Volkmar, F. R. (2010): "Social skills interventions for individuals with autism: Evaluation for evidencebased practices within a best evidence synthesis framework". Journal of Autism and Developmental Disorders, 40 (2): 149-166.

Rivière, Á. y Núñez, M. (2008): La mirada mental. $3^{\text {a }}$ ed. Madrid: AIQUE.

Ruiz de Velasco, Á. y Abad, J. (2011): El juego simbólico. Barcelona: GRAÓ. 
Sancho, K., et al. (2010): "Two Variations of Video Modeling Interventions for Teaching Play Skills to Children with Autism". Education and Treatment of Children, 33 (3): 421-442.

Sani-Bozkurt, S. y Ozen, A. (2015): "Effectiveness and Efficiency of Peer and Adult Models Used in Video Modeling in Teaching Pretend Play Skills to Children with Autism Spectrum Disorder". Education and Training in Autism and Developmental Disabilities, 50 (1): 71-83.

Shukla-Mehta, S. et al. (2010): "Evaluating the Effectiveness of Video Instruction on Social and Communication Skills Training for Children With Autism Spectrum Disorders: A Review of the Literature". Focus on Autism and Other Developmental Disabilities, 25 (1): 23-36.

Ulke-Kurkcuoglu, B. (2015): "A comparison of least-to-most prompting and video modeling for teaching pretend play skills to children with autism spectrum disorder". Kuram ve Uygulamada Egitim Bilimleri, 15 (2): 499-517.

Vigotsky, L. (1978): Pensamiento y lenguaje, en: Obras escogidas Tomo II. Madrid: Machado Libros.

Wang, H. T. y Koyama, T. (2014): "An analysis and review of the literature and a three-tier video modeling intervention model”. Research in Autism Spectrum Disorders, 8 (7): 746-758.

Wert, B. y Neisworth, J. (2003): "Effects of Video Self-Modeling on Spontaneous Requesting in Children with Autism". Journal of Positive Behavior Interventions, 5 (1): 30-34.

Williamson, R. et al. (2012): "Video self-modeling in children with autism: A pilot study validating prerequisite skills and extending the utilization of VSM across skill sets". Assistive Technology, 25: 63-71.

Wilson, K. (2013a): "Incorporating video modeling into a school-based intervention for students with autism spectrum disorders”. Language, Speech, and Hearing Services in Schools, 44 (1): 105-117.

Wilson, K. (2013b): "Teaching social-communication skills to preschoolers with autism: Efficacy of video versus in vivo modeling in the classroom". Journal of Autism and Developmental Disorders, 43 (8): 1819-1831. 